\title{
Forma indeterminada da doença de Chagas: considerações acerca do diagnóstico e do prognóstico
}

\author{
Indeterminate form of Chagas' disease: considerations \\ about diagnosis and prognosis
}

\author{
Antonio Luiz Pinho Ribeiro e Manoel Otávio da Costa Rocha
}

\begin{abstract}
Resumo A forma indeterminada da doença de Chagas é definida pela presença de infecção pelo Trypanosoma cruzi na ausência de manifestações clínicas, radiológicas e eletrocardiográficas de acometimento cardíaco ou digestivo. Pacientes na forma indeterminada podem apresentar anormalidades cardiovasculares significativas à propedêutica mais avançada. Entretanto, a validade do conceito de forma indeterminada tem sido reafirmada, pela simplicidade diagnóstica e benignidade do prognóstico. Na prática clínica, dificuldades diagnósticas são freqüentes, relacionadas à subjetividade e ao significado incerto de achados clínicos, eletrocardiográficos e radiológicos. Adicionalmente, o prognóstico na forma indeterminada não é uniformemente bom: após cinco a 10 anos, postula-se que um terço dos pacientes evoluirão para a forma cardíaca. $A$ morte súbita, uma complicação rara, pode ser a primeira manifestação da doença. É necessária uma reavaliação do conceito de forma indeterminada, com redefinição dos critérios diagnósticos e da conduta terapêutica. A estratificação do risco individual, através de métodos clínicos e nãoinvasivos, pode permitir o reconhecimento de grupos de risco aumentado, passíveis de intervenções terapêuticas. Como o tratamento etiológico pode prevenir o aparecimento da cardiopatia, seu papel no manejo da forma indeterminada deve ser reavaliado.
\end{abstract}

Palavras-chave: Doença de Chagas. Forma indeterminada. Prognóstico. Morte súbita.

Summary The indeterminate form of Chagas' disease is defined by the absence of clinical, radiological and electrocardiographic manifestations of cardiac or digestive involvement in Trypanosoma cruzi chronic infected persons. When submitted to advanced cardiovascular tests, these patients may present significant abnormalities. However, the indeterminate form concept was reaffirmed as valid, since diagnostic criteria are simple and prognosis is benignant. In clinical practice, diagnostic difficulties are frequent, related to subjectivity and uncertain meaning of clinical, electrocardiographic and radiological findings. Moreover, indeterminate form prognosis is not equally good: after five to 10 years, a third of patients will have cardiopathy. Sudden death, a rare complication, may be the first manifestation of Chagas' disease. It is necessary to reappraise indeterminate form concept, redefining diagnostic criteria and therapeutic management. Clinical and noninvasive evaluation may allow individual risk stratification; therapeutic interventions may be beneficial in high risk groups. Since etiologic treatment may prevent cardiopathy, its role in indeterminate form management must be reassessed.

Key-words: Chagas disease. Indeterminate form. Prognosis. Sudden death

Trabalho realizado no Hospital das Clínicas e na Faculdade de Medicina da Universidade Federal de Minas Gerais. Financiado parcialmente pela Fundação de Amparo à Pesquisa de Minas Gerais - FAPEMIG.

Endereço para correspondência: Prof. Antonio Luiz Pinho Ribeiro, Rua Montevidéu, 259/303, Sion, 30315-560 Belo Horizonte, MG. E-mail: antonior@net.em.com.br.

Recebido para publicação em 19/02/97. 
Doença de Chagas e forma indeterminada. A doença de Chagas é um dos principais problemas médico-sociais brasileiros, acometendo atualmente cerca de quatro a seis milhões de pessoas no país 114 . Calcula-se que $8,2 \%$ das 5.074.000 mortes ocorridas no Brasil, no período de 1977 a 1983, foram provocadas por essa enfermidade 116. Por outro lado, a doença de Chagas é uma doença crônica, podendo ser incapacitante e debilitante, com grande impacto sócio-econômico e cultural, sendo a principal causa de aposentadoria precoce em nosso meio114.

De modo geral, reconhece-se a presença de três estádios na doença de Chagas: agudo, crônico indeterminado e crônico determinado 128 . Após período de incubação de cerca de sete a dez dias, inicia-se a fase aguda, geralmente oligossintomática, sendo reconhecida em cerca de, apenas, um a dois por cento dos casos. A doença aguda é mais grave nas crianças menores que dois anos, nas quais, na ausência de tratamento, a letalidade pode chegar a $10 \%$. Após quatro a dez semanas, inicia-se o estádio crônico indeterminado, caracterizado pela ausência de manifestações clínicas, eletrocardiográficas ou radiológicas significativas. Enquanto alguns pacientes permanecem nessa forma indefinidamente, outros, geralmente após intervalo de 10 a 20 anos, evoluem para alguma das formas crônicas determinadas da doença, com aparecimento de evidências de comprometimento cardíaco, digestivo ou neurológico.

Estima-se que cerca de $50 \%$ dos indivíduos infectados se encontrem no estádio indeterminado, ou forma crônica indeterminada da doença $(\mathrm{FCl})$, descrita inicialmente por Carlos Chagas como a ausência das síndromes clínicas predominantes da moléstia26. Em 1984, o conceito de $\mathrm{FCl}$ foi reavaliado por grupo de peritos reunidos em Araxá, MG, tendo sido estabelecidos os seguintes critérios para a sua caracterização 104:

1. positividade sorológica e/ou parasitológica para doença de Chagas;

2. ausência de sintomas e/ou sinais da moléstia;

3. eletrocardiograma convencional normal;

4. estudos radiológicos do coração, esôfago e cólon normais.

Anormalidades cardiovasculares na forma indeterminada da doença de Chagas. Embora a ausência de manifestações clínicas seja a característica mais importante da $\mathrm{FCl}$, sabe-se que, quando estudadas por métodos propedêuticos mais sofisticados, proporção variável de pacientes na $\mathrm{FCl}$ ou sem cardiopatia aparente (ou seja, assintomáticos com eletrocardiograma e radiografia de tórax normais) mostra alterações estruturais ou funcionais do coração e do trato digestivo9 11356592 123. Anormalidades cardiovasculares significativas foram relatadas nestes pacientes através de diferentes métodos não-invasivos:

1. Ergometria: depressão das respostas pressórica e cronotrópica; arritmias ventriculares esforço-induzidas15 51849196112130.

2. Ergoespirometria: diminuição da capacidade funcional máxima72;

3. Eletrocardiografia dinâmica: arritmia ventricular, encontrada em 21 a $69 \%$ dos pacientes chagásicos4 10557983109 123;

4. Vetorcardiografia: 68 a $88 \%$ de estudos anormais, incluindo alterações da repolarização ventricular, sobrecarga ventricular esquerda, transtornos finais da condução, áreas eletricamente inativas, hemibloqueio anterior esquerdo e diminuição das forças septais 74 ;

5. Ecocardiografia: diminuição da fração de encurtamento do ventrículo esquerdo e da velocidade média de encurtamento circunferencial das fibras 4748 ; anormalidades da função diastólica28 318588 e alterações da contractilidade segmentar93 94;

6. Ventriculografia radioisotópica: alterações da contractilidade segmentar à ventriculografia radioisotópica; captação de pirofosfato de tecnécio; defeitos de perfusão à cintilografia de perfusão com tálio; e captação difusa de gálio6 95387123.

7. Provas autonômicas não-invasivas, como a resposta de freqüência cardíaca à arritmia sinusal respiratória, à manobra de Valsalva, à manobra do handgrip e do ortostatismo ativo e a redução de índices vagais da variabilidade da freqüência cardíaca19 565866107108109110111121122.

A presença de anormalidades cardiovasculares estruturais e funcionais foi confirmada em estudos invasivos, como o estudo hemodinâmico20 $21 \quad 33 \quad 52 \quad 76 \quad 82 \quad 113$ e eletrofisiológico16 3334 97. Na verdade, existem evidências anatomopatológicas da presença de alterações estruturais na $\mathrm{FCl}$ da doença de Chagas, incluindo degeneração de miócitos, infiltrado inflamatório e processos fibróticos, lesões dos sistemas de condução e nervoso 
autônomo intracardíaco, obtidos em estudos experimentais em cães 5 e à avaliação de tecido cardíaco humano, obtido através de biópsia endomiocárdica12 2373 ou em corações de pacientes assintomáticos falecidos de modo violento62 6364 .

Entretanto, a interpretação destes achados não é simples. Embora alguns dos trabalhos realizados em pacientes na $\mathrm{FCl}$ tenham sido conduzidos com grande rigor científico, problemas metodológicos relacionados à seleção e ao tamanho das amostras, a ausência de grupo controle simultâneo e a ausência de tratamento estatístico adequado podem ser observados em diversos estudos. Em geral, os autores definem a forma indeterminada sem obediência aos critérios supracitados, selecionando principalmente pacientes com eletrocardiograma normal, com sintomas significativos ou não. Adicionalmente, a interpretação dos achados anatomopatológicos deve considerar as dificuldades metodológicas encontradas: na série de necrópsias realizadas em chagásicos assintomáticos falecidos de modo violento62 63, por exemplo, os autores não dispunham do eletrocardiograma ou radiografia de tórax dos pacientes falecidos. Assim, levando em conta dados epidemiológicos, Lopes e colaboradores 6263 presumiram que $50 \%$ dos pacientes se encontravam na forma indeterminada. Como 27 (90\%) dos 30 pacientes mostravam lesões cardíacas, presumiu-se que no mínimo 12 dos 15 chagásicos assintomáticos apresentavam alterações morfológicas cardíacas.

A presença de alterações aos estudos cardiovasculares não-invasivos também tem sido objeto de controvérsia. Alterações significativas, similares às encontradas em alguns estudos em pacientes com $\mathrm{FCl}$, podem ser encontradas em indivíduos sadios. Como exemplo, sabe-se que ectopia ventricular ao esforço 341 e à monitorização ambulatorial de 24 horas37 59 tem sido relatada na ausência de cardiopatia estrutural. Neste sentido, é significativo que estudos controlados não tenham detectado anormalidades ao teste ergométrico14 4067103106 , à eletrocardiografia dinâmica (Holter de 24h)65 103, ao ecocardiograma 103 e às provas autonômicas 4950 . Quando são encontradas alterações significativas aos métodos propedêuticos cardiológicos, estas geralmente não são indicativas de acometimento cardíaco grave. Entretanto, quando se estudam pacientes na $\mathrm{FCl}$ através de diferentes métodos propedêuticos, praticamente todos eles apresentam pelo menos um exame alterado, e cerca de dois terços apresentam anormalidades em pelo menos três modalidades de exames cardiológicos9. Por este motivo, a $\mathrm{FCl}$ da doença de Chagas tem sido considerada uma "doença polimórfica". O significado prognóstico e evolutivo destas alterações, entretanto, não é conhecido9 333592.

Apesar da presença relativamente freqüente de alterações à propedêutica avançada, o conceito de forma indeterminada tem sido considerado útil, do ponto de vista operacional 35 , já que:

a classificação do paciente nesta forma dependeria apenas da sorologia, de um exame clínico bem realizado, do eletrocardiograma e de exames radiológicos relativamente simples, sendo acessível à maioria dos centros médicos;

os pacientes com $\mathrm{FCl}$ apresentam prognóstico favorável, a médio prazo, e gozam de excelente capacidade laborativa.

Embora as duas afirmações acima estejam embasadas na literatura disponível, o conceito da forma indeterminada apresenta limitações clínicas significativas, como:

dificuldades operacionais no diagnóstico da forma indeterminada; $e$

a constatação de que 2 a $5 \%$ dos pacientes evoluem, ao ano, para formas clínicas definidas da doença de Chagas.

Dificuldades diagnósticas. A simplicidade dos critérios propostos é aparente, já que, na prática, pode ser difícil definir normalidade ao exame clínico, eletrocardiográfico e radiológico sem uma padronização prévia. Freqüentemente, os chagásicos apresentam queixas inespecíficas, de significado controverso, como, por exemplo, dor torácica anginosa atípica80 117118 . O diagnóstico diferencial de outros sintomas, como palpitações e tonteiras, pode exigir a realização de exames específicos, podendo traduzir ou não acometimento cardíaco. Para Manzullo e colaboradores79, "...los síntomas aislados no nos permiten asegurar la presencia de determinada alteración o patología". Desta forma, é usual que pesquisadores classifiquem pacientes como portadores da forma indeterminada, mesmo quando apresentam sintomatologia cardíaca, ressaltando a subjetividade e a inespecificidade de algumas manifestações clínicas54 7998.

A detecção de alterações ao exame físico depende da qualidade técnica do exame e da 
habilidade do examinador. Assim, achados relativamente sutis podem ser marcadores da presença de cardiopatia, embora só sejam reconhecidos quando se realiza exame físico minucioso. Como exemplo, a quarta bulha, indicador de redução da complacência ventricular esquerda, pode passar despercebida para um examinador menos atento, já que em alguns casos só é reconhecida quando se examina o paciente em decúbito lateral esquerdo29. Entretanto, é possível que a quarta bulha seja indicador precoce de dano miocárdico em chagásicos sem cardiopatia107. Por outro lado, a valorização dos achados ao exame físico depende do contexto clínico: a terceira bulha e os sopros sistólicos podem ser achados fisiológicos em adolescentes, mas são marcadores de envolvimento cardíaco em outras situações clínicas29.

Ao eletrocardiograma, alterações relativamente discretas, como a baixa voltagem periférica, o bloqueio incompleto do ramo direito e os bloqueios atrioventriculares de primeiro grau e de segundo grau Mobitz I são descritos tanto em pacientes chagásicos como em indivíduos normais, apresentando potencial evolutivo incerto79. Embora pacientes com tais alterações eletrocardiográficas não sejam incluídos, por definição, na $\mathrm{FCl}$, muitos deles não são cardiopatas. Em algumas circuntâncias clínicas normais, como na vagotonia, alguns destes achados podem ser fisiológicos. Por outro lado, pode-se presumir que algumas alterações, como a baixa voltagem periférica, estejam mais provavelmente relacionadas à existência de dano miocárdico subjacente. Estas nuances não estão previstas no sistema de classificação vigente, de forma que, atualmente, existem pacientes não preenchem critério nem para a forma indeterminada nem para a o diagnóstico de cardiopatia chagásica.

Por fim, a realização dos exames radiológicos contrastados tem benefício questionável para indivíduos assintomáticos. Como o tratamento das lesões digestivas da doença de Chagas está restrito às formas mais avançadas e sintomáticas, é necessário que se reavalie se as informações obtidas através da realização de estudos radiológicos do trato gastrointestinal superam o desconforto e o custo operacional do exame. Na verdade, sabe-se que anormalidades cardiovasculares já foram descritas em pacientes chagásicos não-cardiopatas com manifestações digestivas 69 , incluindo: maior freqüência e gravidade de arritmias ventriculares ao Holter de 24 horas 103;

maior freqüência de arritmias significativas à manobra de Valsalva119;

disfunção autonômica cardíaca caracterizada por diminuição da resposta cronotrópica à manobra de handgrip121 122;

alterações da função ventricular esquerda sistólica e diastólica à avaliação ecocardiográfica ao esforço isométrico120; e

redução da reserva ventricular esquerda avaliada por angiocardiografia nuclear, após administração de isonitrato de isosorbitol81.

Assim, poderia-se supor que a detecção de alterações radiológicas digestivas em chagásicos sem cardiopatia aparente seria importante na detecção de indivíduos com maior incidência de anormalidades cardíacas. Entretanto, a realização de testes cardiovasculares não-invasivos fornece esta mesma informação diretamente e, provavelmente, com melhor relação custobenefício. A não ser que se comprove que o diagnóstico de alterações radiológicas digestivas assintomáticas tem importância no tratamento ou na estratificação de risco de pacientes chagásicos sem cardiopatia aparente, é questionável o valor das informações diagnósticas obtidas pela realização rotineira do estudo contrastado do trato gastrointestinal nestes pacientes.

Implicações prognósticas. O bom prognóstico, a médio prazo, dos pacientes com a FCI da doença de Chagas foi demonstrado por grande número de estudos longitudinais, que confirmaram que as taxas de mortalidade são similares entre pacientes com a $\mathrm{FCl}$ da doença de Chagas e indivíduos não-chagásicos de mesma faixa etária, quando acompanhados por período de três a 10 anos, tanto no Brasil30 364665779098 como também em outros países da América Latina1 2383979102123124 . Vale a pena ressaltar que a maioria destes estudos considera como pacientes com $\mathrm{FCl}$ aqueles nos quais não há cardiopatia, ou apresentam eletrocardiograma normal, não sendo realizado estudo radiológico do trato gastrointestinal.

Embora os pacientes com $\mathrm{FCl}$ da doença de Chagas apresentem, de modo geral, bom prognóstico, cerca de 2 a $5 \%$ destes evoluem anualmente para uma das formas clínicas manifestas da doença35. Sumariando especificamente os diversos estudos longitudinais existentes, avaliando a evolução de pacientes 
com eletrocardiograma normal, Storino et al124 relataram o aparecimento de alterações eletrocardiográficas em 10,4 a 33\% dos pacientes, após cinco anos de seguimento, e em 10 a 48\%, após 10 anos. Dois exemplos significativos, neste sentido, são os trabalhos de Macedo, em São Felipe, Bahia65 e Manzullo e colaboradores, em Buenos Aires, Argentina79.

$\mathrm{Na}$ amostra de São Felipe, Bahia, estudada por Macedo65, verificou-se que $24 \%$ dos pacientes evoluíram para outra forma clínica de doença de Chagas após 10 anos de evolução. Cinqüenta por cento dos que evoluíram apresentavam menos que 20 anos, $40 \%$ estavam entre 20 e 49 anos e apenas 10\% tinham mais de 50 anos. Apenas um indivíduo entre os quatrocentos estudados $(0,25 \%)$ com $\mathrm{FCl}$ faleceu por causa possivelmente associada à doença de Chagas. Sessenta e dois por cento dos pacientes evoluíram para o grau mais leve de cardiopatia, enquanto apenas $7 \%$ evoluíram para as formas mais avançadas.

Em Buenos Aires, na Argentina, Manzullo e colaboradores 79 estudaram 5.710 pacientes com doença de Chagas, dos quais 4.241 foram seguidos por tempo mínimo de um e máximo de 11 anos (média: 5,3 anos). Setenta e seis por cento apresentavam eletrocardiograma normal na primeira consulta. O seguimento destes pacientes por nove anos mostrou que $54 \%$ evoluíram com o aparecimento de alterações eletrocardiográficas. A grande maioria dos pacientes falecidos por causas cardíacas, durante o seguimento, apresentava alterações ao eletrocardiograma na consulta inicial, enquanto que a incidência anual de morte súbita, entre os chagásicos com eletrocardiograma normal, foi baixa: $0,4 \%$.

Muita controvérsia existe sobre quais fatores poderiam influenciar a evolução da doença de Chagas, já que alguns pacientes evoluem para formas mais graves, enquanto outros permanecem assintomáticos por toda a vida. Entre os fatores de risco para o desenvolvimento de complicações cardíacas já foram citados:

sexo masculino 8 36;

cor negra13;

atividade física intensa86;

nível de parasitemia25;

cepa do parasita e fatores geográficos 89 ;

idade e gravidade da infecção aguda inicial36; exposição à reinfecção pelo Trypanosoma cruzi68; história familiar de doença cardiovascular129; estado nutricional, alcoolismo e presença de doenças concomitantes 35 .

Com cinco a 10 anos de evolução, cerca de um terço dos pacientes na $\mathrm{FCl}$ poderão ser cardiopatas 123 . A grande maioria apresentará cardiopatia leve, mas alguns evoluirão para formas mais graves. Embora freqüentemente a cardiopatia tenha curso lento, o prognóstico do paciente cardiopata é significativamente pior do que o da população normal e especialmente sombrio na vigência de insuficiência cardíaca1 3839777990 124. A presença de disfunção ventricular esquerda17 243975 , redução do consumo máximo de oxigênio à ergoespirometria75 e arritmia ventricular complexa ao Holter de 24 horas 24 ou taquicardia ventricular induzida pelo esforço95 estão associados a risco aumentado de morte. Recentemente, evidências iniciais implicaram a redução de índices vagais da variabilidade da freqüência cardíaca 4445126 e a presença de potenciais tardios ao eletrocardiograma de alta resolução 71 como marcadores de risco aumentado de taquicardia ventricular, ou de morte súbita, entre os pacientes chagásicos.

Chagásicos na forma indeterminada morrem subitamente? A morte súbita é o mecanismo de morte em um a dois terços dos pacientes que falecem devido a doença de Chagas30 386899100. Embora a maioria dos pacientes com morte súbita tenha evidências clínicas de insuficiência cardíaca prévia ao evento fatal, cerca de um terço a um quinto das mortes súbitas entre chagásicos ocorrem em pacientes assintomáticos, ocasionalmente sem anormalidades ao exame clínico e radiológico do tórax e, mais raramente, com o eletrocardiograma normal18 101. Assim, a morte súbita pode ser a primeira manifestação clínica da doença de Chagas, retirando a vida de pessoas assintomáticas e produtivas. Chagas e Villela27, em 1922, já haviam reconhecido este fato:

A morte subita constitue o apanagio das regiões de trypanosomíase endêmica. (...)

Falecem os indivíduos, não raro, em plena mocidade e no gozo de uma condição hygida apparente, em phase de tolerancia da affecção cardíaca. Muitos deles morrem no trabalho habitual, sem uma razão immediata que fundamente a occurrencia; outros, porém, veem a fallecer no momento de um maior esforço, de uma fadiga, ou de outro incidente, capaz de esgotar a deficiente energia do myocardio. Os fatos dessa natureza são 
bastante numerosos e evidenciam de sobra a intensidade dos processos pathogenicos da doença. E não sabemos de outra condição, em pathologia humana, que ocasione a morte súbita em percentagem tão elevada quanto o faz a tripanosomiase americana.

Se o prognóstico do chagásico na forma indeterminada é tão favorável, porque parcela significativa dos chagásicos necropsiados por morte súbita não apresentava sintomas ou mesmo alterações à propedêutica cardíaca básica? O caráter retrospectivo do trabalho de Prata et al101 poderia ser citado como causa deste aparente paradoxo: pacientes falecidos subitamente não podem ser entrevistados, e a qualidade da informação que pode ser obtida com os familiares é passível de questionamento. Adicionalmente, nesta série, poucos pacientes necropsiados tinham exames cardiovasculares disponíveis para a análise, de modo que nada impediria que estes pacientes fossem assintomáticos, mas apresentassem evidências marcadas de cardiopatia ao eletrocardiograma ou à radiografia de tórax. Entretanto, o estudo de Bestetti et al 18 , realizado em pacientes com seguimento ambulatorial, incluindo eletrocardiograma e radiografia de tórax, confirma os dados prévios de Prata et al101, indicando que pacientes assintomáticos, sem evidências de cardiopatia à propedêutica básica, podem ter na morte súbita sua primeira manifestação da doença.

Podemos postular, assim, que a morte súbita é uma complicação rara de uma condição comum, a forma indeterminada da doença de Chagas. Existem cerca de dois a três milhões de pacientes na forma indeterminada no Brasil, além de um número adicional significativo de pacientes com cardiopatia silenciosa, assintomáticos ${ }^{114}$. Se considerarmos a incidência de morte súbita como de $0,4 \%$ a a ano, conforme encontrada entre chagásicos sem alterações cardíacas eletrocardiográficas ou radiológicas, no estudo longitudinal de Buenos Aires 79 , podemos esperar mais de mil de óbitos ao ano, na numerosa população brasileira de chagásicos não-cardiopatas. O número de eventos fatais entre pacientes chagásicos assintomáticos, em plena vida ativa, pode ser mais significativo se levarmos em conta que a incidência de morte súbita é maior naqueles pacientes sem sintomas, mas com evidências eletrocardiográficas de cardiopatia1 18777999101. Estudos epidemiológicos longitudinais envolvendo algumas centenas de pacientes não teriam potência suficiente para detectar esta complicação, que só seria reconhecida em estudos prospectivos, envolvendo grande número de pacientes ou em estudos casocontrole rigorosamente desenhados.

De certa maneira, a necessidade de ressaltar o bom prognóstico do chagásico sem cardiopatia, impedindo a estigmatização e a discriminação no mercado de trabalho, pode ter mascarado a importância da morte súbita nestes pacientes: um evento raro, mas que possivelmente acomete número importante de indivíduos, já que existem milhões de chagásicos com formas brandas da moléstia. Por outro lado, a constatação de que cerca de um terço dos pacientes na forma indeterminada apresentarão cardiopatia, após seguimento de cinco a 10 anos ${ }^{124}$, não permite concluir que o prognóstico, para todos estes pacientes, é globalmente favorável. Os pacientes chagásicos na forma indeterminada não se comportam de maneira uniforme ou previsível: existem grupos de risco para morte arrítmica e para evolução para a cardiopatia. Os marcadores que permitiriam reconhecer estes pacientes, entretanto, não se encontram bem estabelecidos.

Perspectivas no manejo clínico do paciente com forma indeterminada. A presente revisão aponta para a pertinência do refinamento do conceito de forma indeterminada da doença de Chagas. É necessária melhor definição dos critérios de normalidade à anamnese, ao exame clínico e à eletrocardiografia, de modo a permitir a caracterização mais homogênea do grupo. Para isto, impõe-se a necessidade do estudo do significado de alterações geralmente pouco valorizadas, mas marcadores potenciais de dano cardíaco, como a quarta bulha ao exame físico. A relação custo-benefício do estudo radiológico contrastado do trato digestivo deve ser considerada: a realização deste está justificada, se a definição da presença de dano digestivo assintomático altera a estratificação de risco destes pacientes ou permite o tratamento preventivo ou curativo de alterações assintomáticas. Caso contrário, acrescenta custo e complexidade à atenção ao chagásico, sem benefício imediato palpável. $\mathrm{O}$ argumento de que a realização do estudo radiológico digestivo estaria indicada para o reconhecimento de pacientes com dano cardíaco incipiente não parece resistir à constatação de que a realização de provas cardiovasculares não-invasivas fornece esta informação de forma direta, com maior precisão e acuidade. 
De maneira análoga, a ergometria, o ecocardiograma, o Holter de 24 horas e as provas autonômicas devem ser realizadas rotineiramente apenas se trouxerem informação relevante para o manejo clínico ou estratificação de risco do chagásico com a forma indeterminada. Neste contexto, a ergometria surge como método privilegiado para o reconhecimento da capacidade laborativa, reproduzindo, sob supervisão médica, o estresse que boa parte dos pacientes é submetido na sua vida cotidiana. A realização deste exame estaria especialmente indicada na avaliação médico-trabalhista ou médico-pericial de indivíduos chagásicos assintomáticos, ou com sintomatologia incaracterística, exercendo atividade que demande esforço extenuante ou continuado, ou atividade de risco.

A ecocardiografia constitui ferramenta útil na avaliação da função ventricular esquerda, permitindo a detecção de alterações da função sistólica, global e regional, da função diastólica, de dilatações ventriculares e atriais e o reconhecimento do aneurisma apical. Embora existam dúvidas quanto a importância evolutiva de índices ecocardiográficos de função sistólica do ventrículo esquerdo, ao modo $M$, no seguimento de pacientes com $\mathrm{FCl} 57$, Cunha encontrou piora progressiva da função diastólica em avaliações seriadas de pacientes na $\mathrm{FCl} 31$. Já o Holter de 24 horas (associado ou não ao estudo da variabilidade da freqüência cardíaca), as provas autonômicas e o eletrocardiograma de alta resolução são métodos de avaliação que, especialmente se realizados conjuntamente, auxiliam no reconhecimento dos pacientes com risco aumentado para morte súbita em diversas cardiopatias $4278125 \mathrm{e}$, possivelmente, na cardiopatia chagásica24 444571 126. Deve-se ressaltar que chagásicos sem cardiopatia podem apresentar alterações ao Holter de 24 horas 107 , à variabilidade da freqüência cardíaca107 $108 \mathrm{e}$ ao eletrocardiograma de alta resolução 70 mesmo na presença de função sistólica do ventrículo esquerdo normal, e na ausência de dilatação ventricular esquerda. Assim, é possível supor que o ecocardiograma seja capaz de identificar pacientes com alterações incipientes da função ventricular esquerda, com potencial evolutivo primariamente para dilatação ventricular e insuficiência cardíaca, enquanto os métodos eletrocardiográficos supracitados sejam capazes de definir um grupo inicialmente com risco arrítmico aumentado e maior possibilidade de evolução para a morte súbita. Com a evolução da doença, disfunção autonômica, arritmia ventricular e disfunção ventricular esquerda parecem interagir de forma sinérgica, de forma que pacientes com piores índices de função ventricular esquerda apresentam arritmia ventricular mais freqüente e complexa22 e disfunção autonômica mais grave32. É necessária, assim, a realização de estudos clínicos, prospectivos e com amostra de tamanho suficiente, para que se defina o papel de cada um destes métodos na estratificação do risco dos pacientes na $\mathrm{FCl}$ da doença de Chagas.

Se o prognóstico do chagásico indeterminado não é tão benigno quanto se imaginava, quais deveriam ser as condutas, diagnósticas e terapêuticas, frente ao paciente nesta condição clínica? Na maioria dos centros de atenção ao chagásico, o cuidado ao paciente na forma indeterminada se resume a orientações gerais e à detecção e ao tratamento sintomático precoce das formas definidas. Entretanto, o grupo populacional com possibilidade de desenvolver uma complicação cardíaca é suficientemente grande para que se estabeleçam estratégias diagnósticas que identifiquem grupos de risco, num processo de "discriminação positiva"61. $\mathrm{Na}$ medida que os fatores determinantes ou que influenciam o aparecimento da cardiopatia são identificados, com quantificação do risco atribuível a cada variável, estratégias no sentido de se eliminar ou controlar cada fator podem ser estabelecidas. Assim, a definição, através de métodos clínicos e complementares, de grupos de risco para a evolução para as formas arrítmica e dilatada da cardiopatia chagásica pode permitir a realização de ensaios terapêuticos randomizados, com medicamentos que atuem sobre a modulação autonômica e arritmogênese, como os betabloqueadores, ou que previnam a dilatação ventricular esquerda, como os inibidores da enzima conversora da angiotensina.

Outra abordagem terapêutica, que não exclui a estratificação de risco discutida acima, tem sido preconizada. Embora ainda prevaleça a recomendação dos peritos reunidos na II Reunião de Pesquisa Aplicada em Doença de Chagas em 1985105, indicando que o tratamento etiológico na $\mathrm{FCl}$ e nas formas definidas incipientes deve ser restrito à investigação clínica, estudos recentes reabriram esta questão. $\mathrm{Na}$ doença de Chagas em crianças, situação na qual o tratamento específico já vinha sendo indicado105, estudo recente indicou para a possibilidade de cura parasitológica. Sgambatti de Andrade e cols115, em ensaio terapêutico controlado e randomizado, avaliaram a eficácia terapêutica do benzonidazol 
em 130 crianças chagásicas entre sete a 12 anos, seguidas por três anos. Após o seguimento, $58 \%$ das crianças tratadas não apresentavam anticorpos contra o Trypanosoma cruzi no sangue periférico, comparado com cinco por cento dos pacientes do grupo placebo. Em adultos na $\mathrm{FCl}$, embora a negativação sorológica ocorra apenas numa minoria dos pacientes 43 , o tratamento etiológico parece ser eficaz na prevenção da progressão da cardiopatia chagásica. Viotti et al127, em ensaio terapêutico controlado em chagásicos seguidos por, em média, oito anos, encontraram que o tratamento específico com benzonidazol diminuiu o aparecimento de novas lesões eletrocardiográficas $(4,2 \%$ versus $30 \%)$ no grupo tratado em relação ao grupo controle, diminuindo também a freqüência de pacientes com deterioração clínica cardíaca $(2,1 \%$ versus $17 \%)$.

"Toute personne infectée par le Trypanosoma cruzi risque de déveloper tôt ou tard une grave complication cardiaque"61. A conduta frente ao chagásico na $\mathrm{FCl}$ não pode continuar sendo a observação passiva dos pacientes, aguardando a instalação da cardiopatia. Estratégias no sentido de se definir grupos de risco, passíveis de intervenção terapêutica e/ou readaptação profissional, devem ser estabelecidas, utilizandose de dados clínicos, epidemiológicos e obtidos através da avaliação cardíaca não-invasiva. Por outro lado, existem evidências significativas de que o tratamento etiológico pode prevenir a progressão da doença de Chagas, indicando que é hora de se redefinir o papel do tratamento específico na $\mathrm{FCl}$.

\section{REFERÊNCIAS BIBLIOGRÁFICAS}

1. Acquatella $H$, Carrasco $H A$, Arribada A, Marinkelle C, Zeledón R, Reyes $P$, Chávez Rivera I. Estudios latinoamericanos. In: Storino RA, Milei J (eds) Enfermedad de Chagas. Inter-médica, Buenos Aires, p. 605-628, 1994.

2. Acquatella H, Catalioti F, Gomez-Mancebo JR, Davalos V, Villalobos L. Long-term control of Chagas' disease in Venezuela: effects on serologic findings, electrocardiographic abnormalities, and clinical outcome. Circulation 76:556-562, 1987.

3. Alfieri RG, Duarte GM. Marcondes. Exercício e o coraçao. $2^{\mathrm{a}}$ ed. Cultura Médica, Rio de Janeiro, 1993.

4. Almeida JWR, Shikanai Yasuda MA, Amato Neto V, Castilho EA, Barretto AC. Estudo da forma indeterminada da doença de Chagas através da eletrocardiografia dinâmica. Revista do Instituto de Medicina Tropical de São Paulo 24:222-228, 1982.
5. Andrade ZA, Andrade SG, Sadigursky M, Maguire $\mathrm{JH}$. Experimental Chagas' disease in dogs. A pathologic and ECG study of the chronic indeterminate phase of the infection. Archives of Pathology and Laboratory Medicine 105:460-464, 1981.

6. Arreaza N, Puigbo JJ, Acquatella $\mathrm{H}$, Casal $\mathrm{H}$, Giordano H, Valecillos R, Mendoza I, Perez JF, Hirschhaut E, Combellas I. Radionuclide evaluation of left-ventricular function in chronic Chagas' cardiomyopathy. The Journal of Nuclear Medicine 24:563-567, 1983.

7. Barretto AC. Aspectos polimórficos da cardiopatia na forma indeterminada da doença de Chagas Estudo através de métodos não invasivos. Tese de Livre-Docência, Faculdade de Medicina da Universidade de São Paulo, 1985.

8. Barretto AC, Arteaga E, Mady C, lanni BM, Bellotti G, Pileggi F. Sexo masculino. Fator prognóstico na doença de Chagas. Arquivos Brasileiros de Cardiologia 60:225-227, 1993.

9. Barretto AC, Azul LG, Mady C, Ianni BM, De Brito Vianna C, Belloti G, Pileggi F. Forma indeterminada da doença de Chagas. Uma doença polimórfica. Arquivos Brasileiros de Cardiologia 55:347-353, 1990.

10. Barretto AC, Bellotti G, Sosa E, Grupi C, Mady C, Ianni BM, Arteaga Fernandez E, Pileggi F. Arritmias e a forma indeterminada da doença de Chagas. Arquivos Brasileiros de Cardiologia 47:197-199, 1986.

11. Barretto AC, Mady C. Forma indeterminada da doença de Chagas. Arquivos Brasileiros de Cardiologia 47:299-302, 1986.

12. Barretto AC, Mady C, Arteaga Fernandez E, Stolf N, Lopes EA, Higuchi ML, Bellotti G, Pileggi F. Right ventricular endomyocardial biopsy in chronic Chagas' disease. American Heart Journal 111:307312, 1986.

13. Baruffa G, Alcantara Filho A, De Aquino Neto JO. Estudo pareado da cardiopatia chagásica no Rio Grande do Sul, Brasil. Comportamento das alteraçoes eletrocardiográficas em função da cor. Memórias do Instituto Oswaldo Cruz 82:399-406, 1987.

14. Bastos A, Manigot DA, Guariento ME, Wanderley J, Santos TC. Eletrocardiograma de repouso/teste cicloergométrico em pacientes com doença de Chagas (forma inaparente). Arquivos Brasileiros de Cardiologia 41 (supl I):85, 1983. 
15. Bellini AJ, Santos RC, Bilac A. Prova de esforço na forma sub-clínica da doença de Chagas. Arquivos Brasileiros de Cardiologia 30:261, 1977.

16. Benchimol CB, Ginefra $P$, Benchimol $A B$. Avaliação eletrofisiológica. In: Cançado JR, Chuster M (eds) Cardiopatia chagásica. Fundação Carlos Chagas, Belo Horizonte, p. 213-222, 1985.

17. Bestetti RB, Dalbo CM, Freitas OC, Teno LA, Castilho OT, Oliveira JS. Noninvasive predictors of mortality for patients with Chagas' heart disease: a multivariate stepwise logistic regression study. Cardiology 84:261-267, 1994.

18. Bestetti RB, Freitas OC, Muccillo G, Oliveira JS. Clinical and morphological characteristics associated with sudden cardiac death in patients with Chagas' disease. European Heart Journal 14:1610-1614, 1993.

19. Caeiro T, losa DJ, Bas J, Palmero HA. Respuesta a la prueba de Valsalva en la enfermedad de Chagas crónica. Medicina (Buenos Aires) 6:767 1978.

20. Carrasco HA. Diagnostico de daño miocardico en la enfermidad de Chagas. Mérida: Universidad de los Andes, 1983.

21. Carrasco HA, Barboza JS, Inglessis G, Fuenmayor A, Molina C. Left ventricular cineangiography in Chagas' disease: detection of early myocardial damage. American Heart Journal 104:595602, 1982.

22. Carrasco HA, Guerrero L, Parada H, Molina C, Vegas E, Chuecos R. Ventricular arrhythmias and left ventricular myocardial function in chronic chagasic patients. International Journal of Cardiology 28:35-41, 1990.

23. Carrasco HA, Palacios Pru E, Dagert de Scorza C, Molina C, Inglessis G, Mendoza RV. Clinical, histochemical, and ultrastructural correlation in septal endomyocardial biopsies from chronic chagasic patients: detection of early myocardial damage. American Heart Journal 113:716-724, 1987.

24. Carrasco HA, Parada H, Guerrero L, Duque M, Duran D, Molina C. Prognostic implications of clinical, electrocardiographic and hemodynamic findings in chronic Chagas' disease. International Journal of Cardiology 43:27-38, 1994.

25. Castro $\mathrm{CN}$. Influência da parasitemia no quadro clínico da doença de Chagas. Revista de Patologia Tropical 9:73-136, 1980.

26. Chagas C, Villela E. Forma cardíaca da Trypanosomiase Americana. Memórias do Instituto Oswaldo Cruz 14:5-61, 1922.
27. Chagas C. Processos patogênicos da Trypanosomiase Americana. Memórias do Instituto Oswaldo Cruz 8:5-37, 1916.

28. Combellas I, Puigbo JJ, Acquatella H, Tortoledo F, Gomez JR. Echocardiographic features of impaired left ventricular diastolic function in Chagas's heart disease. British Heart Journal 53:298-309, 1985.

29. Constant J. Diagnóstico clínico em cardiologia. $3^{\underline{a}}$ edição. Medsi, Rio de Janeiro, 1988.

30. Coura JR, Pereira JB. A follow-up of Chagas' disease in two endemic areas in Brazil. Memórias do Instituto Oswaldo Cruz 79:107-112, 1985.

31. Cunha CLP. Estudo doppler ecocardiográfico evolutivo da função ventricular na forma indeterminada da doença de Chagas. Tese para concurso de Professor Titular. Universidade Federal do Paraná, 1997.

32. Davila DF, Donis JH, Navas M, Feunmayor AJ, Torres A, Gottberg C. Response of heart rate to atropine and left ventricular function in Chagas' heart disease. International Journal of Cardiology 21:143-156, 1988.

33. Décourt LV, Sosa EA, Mady C. Forma indeterminada: conceito e aspectos fisiopatológicos. In: Cançado JR, Chuster M (eds) Cardiopatia chagásica. Fundação Carlos Chagas, Belo Horizonte, p. 121-127, 1985.

34. Décourt LV, Sosa EA, Pileggi F. Estudos eletrofisiológicos cardíacos na forma indeterminada da doença de Chagas. Arquivos Brasileiros de Cardiologia 36:227-234, 1981.

35. Dias JC. The indeterminate form of human chronic Chagas' disease A clinical epidemiological review. Revista da Sociedade Brasileira de Medicina Tropical 22:147-156, 1989.

36. Dias JCP. Doença de Chagas em Bambuí, Minas Gerais, Brasil. Estudo clínico-epidemiológico a partir da fase aguda, entre 1940 e 1982. Tese de Doutorado, Universidade Federal de Minas Gerais, 1982.

37. DiMarco J, Philbrick JT. Use of ambulatory electrocardiographic (Holter) monitoring. Annals of Internal Medicine 113:53-68, 1990.

38. Espinosa R, Carrasco HA, Belandria F, Fuenmayor AM, Molina C, Gonzalez R, Martinez O. Life expectancy analysis in patients with Chagas' disease: prognosis after one decade (1973-1983). International Journal of Cardiology 8:45-56, 1985.

39. Espinosa RA, Pericchi LR, Carrasco HA, Escalante A, Martinez O, Gonzalez R. Prognostic indicators of chronic chagasic cardiopathy. International Journal of Cardiology 30:195-202, 1991. 
40. Faria CAF. Ergometria na avaliação clínica da doença de Chagas crônica. In: Cançado JR, Chuster M (eds) Cardiopatia chagásica. Fundação Carlos Chagas, Belo Horizonte, p. 223-265, 1985.

41. Faris JV, McHenry PL, Jordan JW, Morris SN. Prevalence and reproducibility of exercise-induced ventricular arrhythmias during maximal exercise testing in normal men. American Journal of Cardiology 37:617-622, 1976.

42. Farrell TG, Bashir Y, Cripps T, Malik M, Poloniecki J, Bennett ED, Ward DE, Camm AJ. Risk stratification for arrhythmic events in postinfarction patients based on heart rate variability, ambulatory electrocardiographic variables and the signalaveraged electrocardiogram. Journal of American College of Cardiology 18:687-697, 1991.

43. Ferreira $\mathrm{H}, \mathrm{O}$. Tratamento da forma indeterminada da doença de Chagas com nifurtimox e benzonidazol. Revista da Sociedade Brasileira de Medicina Tropical 23:209-211, 1990.

44. Figueiredo E, Paola AA, Silva RMFL, Távora MZP, Martinez EE. Variabilidade da freqüência cardíaca como preditor de taquicardia ventricular sustentada e morte súbita na cardiopatia chagásica. Arquivos Brasileiros de Cardiologia 66:66, 1996.

45. Figueiredo E, Paola AA, Veloso HH, Silva RMFL, Távora MZP, Martinez EE. Variabilidade da freqüência cardíaca como preditor de taquicardia ventricular sustentada e morte súbita na cardiopatia chagásica. Arquivos Brasileiros de Cardiologia 67:150, 1996.

46. Forichon E. Contribuition aux estimation de morbiditê et de mortalité dans la maladie de Chagas (Trypanosomose américaine). Revista de Patologia Tropical 4:57-78, 1975.

47. Friedmann AA, Armelin E, Nelken JR, Zerbini CA, Coimbra MA, do Serro Azul LG. Estudo ecocardiográfico do desempenho ventricular em fase pré-clínica da doença de Chagas. Revista do Hospital das Clínicas da Faculdade de Medicina de São Paulo 35:165-170, 1980.

48. Friedmann AA. Desempenho ventricular na doença de Chagas. Estudo ecocardiográfico em fase préclínica. Tese de Livre-Docência, Faculdade de Medicina da Universidade de São Paulo, 1978.

49. Fuenmayor AJ, Rodriguez L, Torres A, Donis J, Navas M, Fuenmayor AM, Davila D. Valsalva maneuver: a test of the functional state of cardiac innervation in Chagasic myocarditis. International Journal of Cardiology 18:351-356, 1988.

50. Gallo L Jr, Morelo Filho J, Maciel BC, Marin-Neto JA, Martins LE, Lima Filho EC. Functional evaluation of sympathetic and parasympathetic system in Chagas' disease using dynamic exercise. Cardiovascular Research 21:922-927, 1987.

51. Gallo L Jr, Neto JA, Manco JC, Rassi A, Amorim DS. Abnormal heart rate responses during exercise in patients with Chagas' disease. Cardiology 60:147-162, 1975.

52. Garzon SAC, Lorga AM, Jacob JLB. Forma indeterminada da Doença de Chagas - aspectos hemodinâmicos e cineangiocardiográficos. Revista da Sociedade Brasileira de Medicina Tropical 17:26, 1984.

53. Giorgi MC, Meneguetti JC, Hironaka FH, Barretto AC, Arteaga-Fernandez E, Bellotti G, Pileggi F, Camargo E. Quantificação de captação miocárdica de gálio 67 em pacientes portadores de doença de Chagas. Arquivos Brasileiros de Cardiologia 45:132, 1985.

54. Gontijo ECD. Doença de Chagas transfusional na regiao metropolitana de Belo Horizonte: aspectos clínicos-epidemiológicos e a questão institucional. Tese de Doutorado, Universidade Federal de Minas Gerais, 1989.

55. Gonzalez Zuelgaray J. Eletrocardiografia dinamica y computacion en el estudio de la miocarditis chagasica cronica. In: Posse RA, Mouzo G, Barrio $\mathrm{N}$ (eds) Enfermedad de Chagas. Ministerio de la Salud, Buenos Aires, p. 99-103, 1981.

56. Guzzetti S, losa DJ, Pecis M, Bonura L, Prosdocimi $M$, Malliani A. Impaired heart rate variability in patients with chronic Chagas' disease. American Heart Journal 121:1727-1734, 1991.

57. Ianni BM, Mady C, Barretto ACP, Arteaga E, Bellotti G, Pileggi F. Forma indeterminada da doença de Chagas: avaliacão evolutiva de parâmetros eletrocardiográficos. Arquivos Brasileiros de Cardiologia 67:61, 1996.

58. Junqueira Junior LF, Veiga JPR. Avaliaçao ambulatorial da funçao autonômica cardíaca nas diversas formas clínicas da moléstia de Chagas. Revista da Sociedade Brasileira de Medicina Tropical 17:19, 1984.

59. Kennedy HL, Underhill SJ. Frequent or complex ventricular ectopy in apparently healthy subjects. American Journal of Cardiology 38:141-148, 1976.

60. Köberle F. Chagas' heart disease. Pathology. Cardiology 52:79-96, 1968.

61. Leveque A, De Muynck A. La cardiopathie chagasique chronique: propositions methodologiques pour l'identification es groupes et/ou des comportaments a risque. Médicine Tropicale (Marseille) 53:443-453, 1993. 
62. Lopes ER, Chapadeiro E, Andrade ZA, Almeida $\mathrm{HO}$, Rocha A. Anatomia patologica de chagásicos assintomáticos falecidos de modo violento. Memórias do Instituto Oswaldo Cruz 76:189-197, 1981.

63. Lopes ER, Chapadeiro E, Rocha A. Anatomia patológica do coração na forma indeterminada. In: Cançado JR, Chuster M (eds) Cardiopatia chagásica. Fundação Carlos Chagas, Belo Horizonte, p. 29-40, 1985.

64. Lopes ER, Rocha A, Adad SJ, Fernandes EL, Chapadeiro E. Estudo necroscópico de uma caso da forma crônica da doença de Chagas com eletrocardiograma e raio-X de tórax normais. Referência especial ao sistema excito condutor do coração. Revista da Sociedade Brasileira de Medicina Tropical 21:67-70, 1988.

65. Macedo V, Praga A, Silva A. Teste da pilocarpina na forma indeterminada da doença de Chagas. Revista Goiana de Medicina 20:191-199, 1974.

66. Macedo V, Santos R, Prata A. Prova de esforço na forma indeterminada da doença de Chagas. Revista da Sociedade Brasileira de Medicina Tropical 7:313-317, 1973.

67. Macedo V. Forma indeterminada da doença de Chagas. Jornal Brasileiro de Medicina 38:34-40, 1980.

68. Macedo VO. Influência da exposição à reinfecção na evolução da doença de Chagas. Tese de Docência livre, Universidade Federal do Rio de Janeiro, 1973.

69. Maciel BC, Almeida Filho OC, Schmidt A, MarinNeto JA. Função ventricular na moléstia de Chagas. Revista da Sociedade de Cardiologia do Estado de São Paulo 4:144-151, 1994.

70. Madoery C, Guindo J, Madoery RJ. Eletrocardiografía de alta resolución: potenciales ventriculares tardios en la enfermedad de Chagas. In: Madoery RJ, Madoery C, Cámera MI (eds) Actualizaciones en la enfermedad de Chagas. Organismo Oficial del Congresso Nacional de Medicina, Buenos Aires, p. 149-162, 1993.

71. Madoery C, Ruiz A, Scaglione J, Socas A, Tentori C, Madoery R, Cámera MC. Are the signalaveraged ECG in chagasic patients an important tool? European Journal of Cardiac Pacing and Electrophysiology 6:104 1996.

72. Mady C, Barretto AC, lanni BM, Lopes EA, Pileggi F. Right ventricular endomyocardial biopsy in undetermined form of Chagas' disease. Angiology 35:755-759, 1984.
73. Mady C, Barretto AC, Moffa PJ, Ianni BM, Arteaga Fernandez E, Belotti G, Pileggi F. $O$ vectorcardiograma na forma indeterminada da doença de Chagas. Arquivos Brasileiros de Cardiologia 44:83-85, 1985.

74. Mady C, Cardoso RHA, Barretto ACP, Luz PL, Bellotti G, Pileggi F. Survival and predictors of survival in patients with congestive heart failure due to Chagas'cardiomyopathy. Circulation 90:30983102, 1994.

75. Mady C, de Moraes AV, Galiano N, Decourt LV. Estudo hemodinâmico da forma indeterminada da doença de Chagas. Arquivos Brasileiros de Cardiologia 38:271-275, 1982.

76. Mady C. Estudo da capacidade funcional máxima pela ergoespirometria em pacientes portadores de doença de Chagas. Tese de Livre-Docência, Faculdade de Medicina da Universidade de São Paulo, 1985.

77. Maguire JH, Hoff R, Sherlock I, Guimaraes AC, Sleigh AC, Ramos NB, Mott KE, Weller TH. Cardiac morbidity and mortality due to Chagas' disease: prospective electrocardiographic study of a Brazilian community. Circulation 75:1140-1145, 1987.

78. Malik M, Camm AJ. Heart rate variability and clinical cardiology. British Heart Journal 71:3-6, 1994.

79. Manzullo EC, Darraidou MA, Libonatti O, Rozlosnik J, Bazzano AC. Estudio longitudinal de la cardiopatia chagasica cronica. Centro de Chagas de la Catedra de Enfermedades Infecciosas de la Facultad de Ciencias Medicas de Buenos Aires, 1982.

80. Marin-Neto JA, Marzullo P, Marcassa C, Gallo Junior L, Maciel BC, Bellina CR, L'Abbate A. Myocardial perfusion abnormalities in chronic Chagas' disease as detected by thallium-201 scintigraphy. American Journal of Cardiology 69:780-784, 1992.

81. Marin-Neto JA, Sousa AC, Maciel BC, Gallo Junior $\mathrm{L}$, lazigi N. Avaliação angiocardiográfica nuclear do efeito do dinitrato de isosorbitol em pacientes chagasicos. Arquivos Brasileiros de Cardiologia 51:367-371, 1988.

82. Marins N, da Silva CT, da Motta VP, Scianni CC, Arcaldi N, de Matos JV. Estudo hemodinâmico em indivíduos chagásicos sem cardiopatia aparente. Arquivos Brasileiros de Cardiologia 37:463-466, 1981.

83. Marins N, Flores AP, Seixas TN, da Costa Fagundes J, Ostrowsky M, De Marco Martins A, 
Franco PR. Estudo da forma indeterminada da doença de Chagas através da eletrocardiografia dinâmica. Arquivos Brasileiros de Cardiologia 39:303-307, 1982

84. Marins N, Seixas TN, Lobo V, Flores AP, Miranda LF, Marins AM. Teste Ergométrico nas formas digestiva e indeterminada da doença de Chagas. In: Araújo WB (ed) Ergometria e cardiologia desportiva. Medsi, Rio de Janeiro, p.196-224, 1986.

85. Martinez Filho OR, Carrasco H, Molina CA, Mendez M. Estudio de la función diastólica ventricular izquierda en pacientes com enfermedad de Chagas. Arquivos Brasileiros de Cardiologia 47:3136, 1986.

86. Mathews JC. Valor de la prueba de esfuerzo graduado (ergometria) para determinar la capacidad laboral del cardiopata cronico chagasico. Tesis de Doctorado, Universidad Nacional de Cordoba, 1973.

87. Meneghelo RS, Thom AF, Martins LR. Aspectos da cintilografia miocárdica. In: Cançado JR, Chuster M (eds) Cardiopatia chagásica. Fundação Carlos Chagas, Belo Horizonte, p. 184-188, 1985.

88. Migliore RA, Guerrero FT, Armenti A, Fernandez C, Adaniya ME, lannariello J, Tamagusuku $\mathrm{H}$, Mouzo G, Baudino C, Posse R. Funcion diastolica en la enfermedad de Chagas. Medicina (Buenos Aires) 50:537-542, 1990.

89. Miles MA, Cedillos RA, Povoa MM, de Souza AA, Prata A, Macedo V. Do radically dissimilar Trypanosoma cruzi strains (zymodemes) cause Venezuelan and Brazilian forms of Chagas' disease? The Lancet 1:1338-1340, 1981.

90. Mota EA, Guimaraes AC, Santana OO, Sherlock I, Hoff R, Weller TH. A nine year prospective study of Chagas' disease in a defined rural population in northeast Brazil. The American Journal of Tropical Medicine and Hygiene 42:429-440, 1990.

91. Oliveira Jr WA, Salazar LF, Malta J, Assi N. Análise crítica da forma indeterminada da doença de Chagas. Arquivos Brasileiros de Cardiologia 47:283-288, 1986.

92. Oliveira Jr WA. Forma indeterminada da doença de Chagas. Avaliação através do teste ergométrico. Tese de Mestrado, Universidade Federal de Pernambuco, 1987.

93. Ortiz J, Barretto AC, Matsumoto AY, Monaco CA, lanni B, Marotta RH, Mady C, Bellotti G, Pileggi F. Alteraçäo contrátil segmentar na forma indeterminada da Doença de Chagas. Estudo ecocardiográfico. Arquivos Brasileiros de Cardiologia 49:217-220, 1987.
94. Ortiz J. Aspectos ecocardiográficos. In: Cançado JR, Chuster M (eds) Cardiopatia chagásica. Fundação Carlos Chagas, Belo Horizonte, p. 165168, 1985.

95. Paola AA, Gomes JA, Terzian AB, Miyamoto $\mathrm{MH}$, Martinez Filho EE. Ventricular tachycardia during exercise testing as a predictor of sudden death in patients with chronic chagasic cardiomyopathy and ventricular arrhythmias. British Heart Journal 74:293-295, 1995.

96. Pereira MH, Brito FS, Ambrose JA, Pereira CB, Levi GC, Neto VA, Martinez EE. Exercise testing in the latent phase of Chagas' disease. Clinical Cardiology 7:261-265, 1984.

97. Pimenta J, Miranda M, Pereira CB. Electrophysiologic findings in long-term asymptomatic chagasic individuals. American Heart Journal 106:374-380, 1983.

98. Pompeu FR. Estudo longitudinal da doença de Chagas em trabalhadores rurais do município de Luz, Minas Gerais (1976-1985). Tese de Mestrado, Universidade Federal de Minas Gerais, 1990.

99. Porto CC. O eletrocardiograma no prognóstico e evolução da doença de Chagas. Arquivos Brasileiros de Cardiologia 17:313-346, 1964.

100. Prata A, Lopes ER, Chapadeiro E. Características da morte súbita tida como inesperada na doença de Chagas. Revista da Sociedade Brasileira de Medicina Tropical 19:9-12, 1986.

101. Prata A, Lopes ER, Chapadeiro E. Morte súbita. In: Cançado JR, Chuster M (eds) Cardiopatia chagásica. Fundação Carlos Chagas, Belo Horizonte, p. 114-120, 1985.

102. Puigbó JJ, Nava Rhode JR, Garcia Barrios H, Gil Yepez C. Quatro años de estudio de una comunidad rural con endemicidad chagasica. Boletín de la Oficina Sanitaria Panamericana 66:112-120, 1969.

103. Rassi A, Rassi Jr A, Rassi AG, Rassi Jr L, Rassi $S G$. Avaliação da forma indeterminada da doença de Chagas através de métodos não invasivos. Arquivos Brasileiros de Cardiologia 57:C140, 1991.

104. I Reunião de Pesquisa Aplicada em Doença de Chagas. Validade do conceito de forma indeterminada de doença de Chagas. Revista da Sociedade Brasileira de Medicina Tropical 18:46, 1985.

105. II Reunião de Pesquisa Aplicada em Doença de Chagas. Tratamento específico. Revista da Sociedade Brasileira de Medicina Tropical 19:102103, 1986. 
106. Ribaute E, Lemesre JL, Rodriguez C, Carrasco R, Breniere F, Antezana G, Raynaud J, Carlier Y. Bioenergetic and cardiovascular responses to exercise in residents at $2.850 \mathrm{~m}$, with asymptomatic Chagas' disease. Tropical and Geographical Medicine 38:150-157, 1986.

107. Ribeiro ALP, Moraes RS, Torres RM, Ferlin EL, Oliveira E, Rohde LEP, Gontijo ECD, Ribeiro JP, Rocha MOC. Reduced heart rate variability preceding left ventricular dilatation in Chagas disease. European Journal of Cardiac Pacing and Electrophysiology 6:106, 1996.

108. Ribeiro ALP, Moraes RS, Torres RM, Tostes VTV, Abreu CDG, Ferlin EL, Oliveira E, Rohde LEP, Ribeiro JP, Rocha MOC. Arritmia ventricular e disfunção autonômica ocorrem precocemente na doença de Chagas. Arquivos Brasileiros de Cardiologia 67:105, 1996.

109. Ribeiro ALP, Rocha MOC, Torres RM, Gontijo ECD, Ferreira LM, Oliveira E, Silva FA. Resposta ao estresse ortostático ativo em chagásicos sem cardiopatia aparente. Revista da Sociedade Brasileira de Medicina Tropical 27:147-148, 1994.

110. Ribeiro ALP, Rocha MOC, Torres RM, Gontijo ECD, Ferreira LM, Oliveira E, Silvério MJB. Disfunção autonômica detectada através do teste da arritmia sinusal respiratória em chagásicos sem cardiopatia aparente. Arquivos Brasileiros de Cardiologia 63:88, 1994

111. Ribeiro ALP, Tostes VTV, Torres RM, Abreu CDG, Couto MOR, Gontijo ECD, Rocha MOC. Teste ergométrico em chagásicos sem cardiopatia aparente. Arquivos Brasileiros de Cardiologia 65:96, 1995.

112. Ribeiro ALP. Disfunçao autonômica e arritmia ventricular em chagásicos sem cardiopatia aparente. Tese de Doutorado, Faculdade de Medicina da Universidade Federal de Minas Gerais, 1996.

113. Saad EA, Salles Netto M, Pryzytyk RF, Feres JGF, Argueles E, Abraão C. Estudo hemodinâmico. In: Cançado JR, Chuster M (eds) Cardiopatia chagásica. Fundação Carlos Chagas, Belo Horizonte, p. 188-212, 1985.

114. Schmunis GA. American Trypanosomiasis as a public health problem. In: Pan American Health Organization (ed) Chagas' Disease and the nervous system. Pan American Health Organization, Washington, DC, p. 3-29, 1994.

115. Sgambatti de Andrade ALS, Zicker F, Oliveira RM, Almeida e Silva S, Luquetti A, Travassos LR, Almeida IC, Andrade SS, Andrade JG, Martelli
CMT. Randomised trial of efficacy of benznidazole in treatment of early Trypanosoma cruzi infection. The Lancet 348:1407-1413, 1996.

116. Silveira AC. Mortalidade por doença de Chagas no Brasil, 1977/1983. Memórias do Instituto Oswaldo Cruz 81:70, 1986.

117. Simoes MV, Ayres EM, Santos JL, Schmidt A, Pintya AO, Maciel BC, Marin-Neto JA. Detecção da isquemia miocárdica em chagásicos crônicos precordialgia atípica pelos testes de esforço e Holter. Arquivos Brasileiros de Cardiologia 60:315319, 1993.

118. Simoes MV, Dantas RO, Ejima FH, Meneghelli UG, Maciel BC, Marin-Neto JA. Origem esofágica de dor precordial em pacientes chagasicos com artérias coronárias subepicárdicas normais. Arquivos Brasileiros de Cardiologia 64:103-108, 1995.

119. Soares JD, Junqueira Junior LF. Incidência de arritmias associadas à manobra de Valsalva nas diversas formas clínicas da doença de Chagas. Revista da Sociedade Brasileira de Medicina Tropical 17:58, 1984.

120. Sousa AC, Marin-Neto JA, Maciel BC, Gallo Junior L, Amorim D, S, Barreto Martins LE. Disfunção sistólica e diastólica nas formas crônica indeterminada, digestiva e cardíaca da doença de Chagas. Arquivos Brasileiros de Cardiologia 50:293-299, 1988.

121. Sousa AC, Marin-Neto JA, Maciel BC, Gallo Junior L, Amorim DS. Cardiac parasympathetic impairment in gastrointestinal Chagas' disease. The Lancet 1:985, 1987.

122. Sousa AC, Marin-Neto JA, Maciel BC, Gallo Junior L, Barreto Martins LE, Amorim DS. Use of isometric exercise to demonstrate cardiac parasympathetic impairment in the digestive form of Chagas' disease. Brazilian Journal of Medical and Biological Research 20:781-783, 1987.

123. Storino R, Milei J, Manzullo E, Darraidou M. Evolucion natural y estudios longitudinales. In: Storino RA, Milei J (eds) Enfermedad de Chagas. Inter-médica, Buenos Aires, p. 593-604, 1994.

124. Storino R. Chagas indeterminado. In: Storino RA, Milei J (eds) Enfermedad de Chagas. Inter-médica, Buenos Aires, p. 235-246, 1994.

125. Task Force of the European Society of Cardiology and the North American Society of Cardiac Pacing and Electrophysiology. Heart rate variability. Standarts of measurement, physiological interpretation, and clinical use. Circulation 93:10431065, 1996. 
126. Veloso HH, Paola AA, Figueiredo E, Silva RMFL, Távora MZP, Martinez EE. Comparação da variabilidade da freqüência cardíaca no domínio do tempo entre pacientes chagásicos com taquicardia ventricular sustentada e não sustentada e frações de ejeção semelhantes. Revista da Sociedade de Cardiologia do Estado de São Paulo 6:24, 1996.

127. Viotti R, Vigliano $\mathrm{C}$, Armenti $\mathrm{H}$, Segura E. Treatment of chronic Chagas' disease with benznidazole: clinical and serologic evolution of patients with longterm follow-up. American Heart Journal 127:151162, 1994.

128. World Health Organization Expert Committee on the Control of Chagas Disease. Control of Chagas
Disease. Geneva: World Health Organization. WHO technical reports series: 811, p.1, 1991.

129. Zicker F, Smith PG, Netto JC, Oliveira RM, Zicker EM. Physical activity, opportunity for reinfection, and sibling history of heart disease as risk factors for Chagas' cardiopathy. American Journal of Tropical Medicine and Hygiene 43:498-505, 1990.

130. Zicker F, Zicker EM, Oliveira JJ, Netto JC, Oliveira RM, Smith PG. Exercise electrocardiogram tests in manual workers with and without antibodies to Trypanosoma cruzi: a population-based study. Transactions of the Royal Society of Tropical Medicine and Hygiene 84:787-791, 1990. 